\title{
Programa convivimos en armonía para mejorar las habilidades sociales en la institución educativa 82210 Nuevo Chao - Virú, 2021
}

\author{
Annie Carolina Culcas Taboada \\ carolina19ct@gmail.com \\ https://orcid.org/0000-0003-0959-6755 \\ Universidad Cesar Vallejo \\ Trujillo - Peru
}

\section{RESUMEN}

La presente investigación tuvo como objetivo. Demostrar de qué manera el Programa convivimos en armonía influirá en la mejora de las habilidades sociales en estudiantes del sexto grado de primaria de la Institución Educativa N ${ }^{\circ} 82210$ Nuevo Chao -Virú. Además, proponer un programa sobre convivencia para mejorar las habilidades sociales. La investigación es de tipo aplicada, y con diseño cuasi experimental. Se aplicó un cuestionario sobre habilidades sociales a una muestra de 60 estudiantes de educación primaria seleccionados probabilísticamente. Tal como se muestra en la Dimensión Interacción Social 26,7\%, Concepción de amigos 26,7\%, Desarrollo de Sentimientos y emociones 23,3\%, Relaciones interpersonales 23,3\%.Los resultados de la aplicación del post test revelan que el Programa Convivimos En Armonía ha contribuido en el desarrollo de Habilidades Sociales en los estudiantes de la Institución Educativa cuyos porcentajes alcanzados son:Dimensión Interacción Social 40\%,Concepción de amigos 40\%,Desarrollo de Sentimiento y emociones 36.7\%, Interpersonal 50\%. Así mismo, se obtuvo el siguiente resultado de la prueba de normalidad "Shapiro Wilk" $(\mathrm{n}<50)$, con el nivel de significación del 0.05, la misma que arroja un sig $<0,05$ en cada uno de los factores utilizados en la investigación. Concluyéndose que el programa convivimos en armonía si influye en la variable sobre habilidades sociales. Finalmente, se presentó como propuesta el programa convivimos en armonía para mejorar las habilidades sociales con 6 características esenciales como: Interacción social, concepción de amigos, desarrollo de emociones y sentimientos, relaciones interpersonales.

Palabras clave: emociones; habilidades sociales; interacción social 


\title{
We coexist in harmony program to improve social skills at educational institution 82210 Nuevo Chao - Virú, 2021
}

\begin{abstract}
The present investigation had as objective. Demonstrate how the Program We Coexist in Harmony will influence the improvement of social skills in sixth grade students of the Educational Institution N 82210 Nuevo Chao -Virú. In addition, propose a program on coexistence to improve social skills. The research is of an applied type, and with a quasiexperimental design. A questionnaire on social skills was applied to a sample of 60 probabilistically selected primary school students. As shown in the Social Interaction Dimension $26.7 \%$, Conception of friends $26.7 \%$, Development of feelings and emotions $23.3 \%$, Interpersonal relationships $23.3 \%$. The results of the application of the post-test reveal that the The Convivimos En Armonía Program has contributed to the development of Social Skills in the students of the Educational Institution whose percentages reached are: Social Interaction Dimension 40\%, Conception of friends 40\%, Development of Feelings and emotions $36.7 \%$, Interpersonal 50\%. Likewise, the following result of the "Shapiro Wilk" test of normality was obtained ( $\mathrm{n}<50$ ), with a significance level of 0.05 , the same that yields a sig $<0.05$ in each of the factors used in the Research, concluding that the program we live in harmony if it influences the variable on social skills. Finally, the program we coexist in harmony was presented as a proposal to improve social skills with 6 essential characteristics such as: Social interaction, conception of friends, development of emotions and feelings, interpersonal relationships.
\end{abstract}

Keywords: emotions, social skills, social interaction

Artículo recibido: 02 noviembre. 2021 Aceptado para publicación: 28 noviembre 2021 Correspondencia: carolina19ct@gmail.com Conflictos de Interés: Ninguna que declarar 
Culcas Taboada...

\section{INTRODUCCIÓN}

Los humanos por naturaleza somos seres sociales, relacionarnos y comunicarnos con los demás es fundamental en nuestras vidas, porque nos va a permitir convivir de manera armónica, compartiendo experiencias, si somos asertivos los conflictos y malos entendidos se solucionarán sin ninguna dificultad. y Las habilidades sociales y el clima social familiar son procesos que se desarrollan y adquieren en cada familia, la relación que haya entre ellos es un factor clave, aquí el niño aprende conductas de cada uno de los miembros y los lleva a la práctica, donde seleccionará las que crea conveniente.

Si estas habilidades están ausentes podemos caer en diferentes situaciones, de las cuales no podremos salir tan fácilmente, generando conflictos pasivos y/o agresivos. Debemos tener en cuenta que cada persona aprende de diferente manera, si a esto le añadimos habilidades sociales se formaran un vigoroso eje de de aprender a aprender y ajuste social. Son muchos los beneficios que adquirimos cuando ponemos en práctica las habilidades sociales nos ayudará a lograr nuestros objetivos y a comunicarnos de una manera empática con las demás personas, regula aprendizajes, influye en los demás, mejorando nuestra autoestima, asertividad y disminuyendo el estrés.

La UNESCO considera que en el siglo XXI se debe complementar diferentes habilidades como el aprendizaje informal, colaboración, comunicación destacando las competencias sociales y competencias personales como esenciales para vivir en sociedad y destacar en el ámbito laboral.

En las diferentes instituciones educativas podemos darnos cuenta la ausencia de habilidades sociales, ocurren entercados con niños del mismo ciclo y de ciclos diferentes, los estudiantes no se respetan, insultan y agreden a sus compañeros, no respetan las autoridades de la escuela, en las calles no respetan a los ancianos, insultan a todo el que se le antepone en el camino, la escases de comunicación dentro del entorno familiar, y los aprendizajes del entorno social ha influido a que los niños como personas vulnerables adquieran conductas que causan malestar al momento de relacionarse.

El éxito en la vida, felicidad y logros, no solo es adquirir habilidades cognitivas, es desarrollar a gran complejidad habilidades sociales, desarrollar relaciones interpersonales y habilidades de interactuar con otras personas, al lograr esto nos permitirá tener una confianza en nosotros mismos. En las escuelas le dan más importancia al aspecto cognitivo, dejando de lado la parte humana, siendo este último muy importante para una 
convivencia armónica. Las habilidades sociales van determinar una paz y tranquilidad en los seres humanos nos ayudaran a respetarnos entre nosotros, a ser empáticos, asertivos buscando el bienestar de las demás personas que nos rodean, nos sentiremos bien por tales comportamientos, estas habilidades la mayoría de veces son aprendidas, desarrolladas y luego llevadas a la práctica, algunas de las habilidades sociales son sencillas y otras son complejas; de acuerdo a como lo apliquemos iremos reforzándolo.

La clave para poder solucionarlo de manera imparcial, es detectar el origen de estas discusiones, centrarnos en buscar soluciones mediante la detección del protagonista, conversar con ambas partes y buscar entre ellos soluciones que sean justas, esto se dará a partir de la argumentación.

Los niños requieren desplegar una complejidad de habilidades para manejar el estrés tóxico y perfeccionar su desarrollo. Diferentes investigaciones señalan que para el desarrollo con padres y compañeros el juego apropiado es una excelente estrategia para lograr desarrollar autocontrol y habilidades cognitivas del lenguaje que desarrollan la función ejecutiva y un cerebro prosocial. Además, el juego forma parte de una destacada relación estable, seguras y enriquecedora, genera en los niños prosperidad en relaciones sociales.

Es importante que el niño, realice una serie de juegos, para desarrollar habilidades presociales lo cuales permitirán disminuir el estrés, lograra generar seguridad y reacciones amicales muy buenas.

Como docentes tenemos una gran responsabilidad para que nuestros estudiantes adquieran estas habilidades; nuestro trabajo va más allá de detectar, es cómo mejorar y lograr que las personas que pertenecen al entorno educativa se empoderen y lo apliquen en su día a día

\section{ESTRATEGIAS METODOLÓGICAS O MATERIALES Y MÉTODOS}

\subsection{Tipo y diseño de investigación}

El enfoque asumido fue el cuantitativo. El tipo de estudio de la presente investigación fue aplicado y orientado a la Implementación de un Programa "convivimos en armonía" para desarrollar las habilidades sociales en nuestros estudiantes.

En la presente investigación utilizó el diseño cuasiexperimental, de grupo único con PreTest y Post- Test. 
\begin{tabular}{ccc}
$G E: 0_{1}$ & $X$ & $0_{2}$ \\
\hdashline$C: 0_{3}$ & & $0_{4}$
\end{tabular}

\section{Donde:}

M: muestra

O1: Pre test

O2: Post test

X: Estímulo: Programa "convivimos en armonía"

\subsection{Variables y operacionalización:}

En esta investigación la variable y sus respectivas dimensiones fueron las siguientes:

- Variable independiente: Programa convivimos en armonía.

Dimensiones: instrucciones, modelado o aprendizaje por observación, representación de los papeles o Role- playing:,retroalimentación o feedback, reforzamiento, tareas para casa.

\section{- Variable dependiente: Habilidades sociales}

Dimensiones: interacción social, concepción de amigos, desarrollo de emociones y sentimientos, relaciones interpersonales

\subsection{Población, muestra y muestreo:}

La población objeto de estudio del presente trabajo estará comprendido por 150 estudiantes de sexto grado de educación primaria de la Institución Educativa 82210 Nuevo Chao - Virú, 2021

\section{Tabla 01}

Distribución de los estudiantes de sexto de primaria.

Población de estudio

\begin{tabular}{|c|c|c|c|}
\hline Grado & Sección & Cantidad & Porcentaje \\
\hline $6^{\circ}$ & $\bar{A}$ & 30 & $20 \%$ \\
\hline $6^{\circ}$ & B & 30 & $20 \%$ \\
\hline $6^{\circ}$ & $\mathrm{C}$ & 30 & $20 \%$ \\
\hline $6^{\circ}$ & $\mathrm{D}$ & 30 & $20 \%$ \\
\hline $6^{\circ}$ & E & 30 & $20 \%$ \\
\hline \multicolumn{2}{|c|}{ Total } & 150 & $100 \%$ \\
\hline
\end{tabular}

Fuente: Nómina de la I.E. 82210 Karina Damian Violeta 


\section{Criterios de selección}

Con el propósito de asegurar resultados verídicos y realistas de la aplicación del Programa "Convivimos en armonía", se estableció una serie de criterios de inclusión y exclusión para la muestra de investigación.

\section{Criterios de inclusión}

Se consideró a los estudiantes de sexto grado de primaria de las secciones A y B por tener la misma cantidad de estudiantes y condiciones similares como: el mismo turno de estudio, porque pertenecen al mismo intervalo de edad comprendidas entre los 11 y 12 años, con sexos diferentes, cuya clasificación socioeconómica oscila entre el no pobre y pobre; con diferentes estilos de aprendizaje, asistencia permanente e interés voluntario a participar.

\section{Criterios de exclusión}

No se consideró a los estudiantes que tiene problemas de conectividad, pues se tipifica como ausentismo escolar.

No se consideró a los estudiantes que se encuentran enfermos o con alguna dispensa oficial.

No se consideró a los estudiantes que se encuentran en proceso de traslado.

No se consideró a los estudiantes con asistencia intermitente.

No se consideró a los estudiantes con necesidades educativas especiales.

Unidad de análisis: Cada uno de los estudiantes de sexto grado de educación primaria de una institución educativa pública de Virú

Muestra y muestreo. La muestra de estudio se estimó a través del muestreo aleatorio simple conformada por estudiantes cuarto grado de educación primaria de la Institución Educativa 82210 del distrito de Nuevo Chao, habiéndose obtenido como tamaño muestral 30 para cada grupo según como consta en el anexo $\mathrm{N}^{\circ} 07$ y en la siguiente tabla:

\section{Tabla 2}

Muestra de estudio

\begin{tabular}{lcc}
\hline Grupo de Investigación & Estudiantes & Porcentaje \\
\hline Grupo Control & 30 & $50 \%$ \\
\hline Grupo Experimental & 30 & $50 \%$ \\
\hline Total & 60 & $100 \%$ \\
\hline
\end{tabular}

Fuente: Nómina de la I.E. 82210 Karina Damian Violeta 
Culcas Taboada...

\subsection{Técnicas e instrumentos de recolección de datos,}

Para la recolección y análisis de datos en esta investigación se utilizó las siguientes técnicas e instrumentos:

\subsubsection{Técnicas. e instrumentos}

\section{Técnicas}

Para la variable dependiente denominada Habilidades sociales se utilizó una encuesta para obtener y registrar los datos de la muestra de estudio.

Para la variable independiente denominada programa convivimos en armonía se aplicó la técnica de la observación, la cual permitió dar seguimiento a la ejecución del programa en la muestra de estudio.

\section{Instrumentos}

Para la variable dependiente, la autora elaboró un cuestionario de habilidades sociales escrita a manera formulario google. Estuvo compuesta por 20 preguntas tipo ensayo, la misma que fue aplicada a cada uno de los estudiantes que conformaron la muestra de estudio. Esta prueba se aplicó en el pre y post test, tanto al grupo experimental como al grupo control. Los ítems estuvieron organizados según las 4 dimensiones de la siguiente manera: para la dimensión Interacción social le corresponden el ítem 1,2,3,4 y 5 para la dimensión concepción de amigos le corresponde los ítems 6,7,8,9 y 10 mientras que para la dimensión desarrollo de emociones y sentimientos le corresponde los ítems 11,12,13,14 y 15. La escala utilizada fue cuantitativa discreta presentada y valorada de la siguiente manera: Alto, medio, y bajo.

- Para la variable independiente, la autora elaboró un programa con 15 sesiones, en función de las 6 dimensiones del programa convivimos en armonía, las dimensiones se aplicarán durante toda las sesión de aprendizaje; las dimensiones son: Instrucciones, Modelado o aprendizaje por observación, representación de los papeles o Role- playing, Retroalimentación o feedback, Reforzamiento, Tareas para casa. Dicho instrumento fue aplicado indirectamente al grupo experimental. Asimismo, los resultados fueron presentados de forma cualitativa en el orden siguiente: alto=8-10, medio $=5-7$, bajo $=0-4$.

\subsubsection{Validez y confiabilidad de los instrumentos}




\section{El presente se obtuvo}

La validez del instrumento se realizó a través de 5 expertos, Así mismo, se utilizó la V de Aiken para determinar el índice de validez, siendo ese v=1.0, lo que significa que el instrumento es válido en lo que corresponde a pertinencia, relevancia y claridad. En relación a la variable y su respectivo dimensiones

En cuanto a la confiabilidad, se aplicó un sondeo piloto con 20 estudiantes ajenos al grupo de investigación, pero de similares características.

Luego, utilizando el programa SPPS24, se determinó mediante el estadístico alfa de cronbach resultado como valor alfa $=0,489$, lo que nos indica que el instrumento es altamente confiable para obtener datos objetivos y constantes en relación a la variable.

Tabla 3 Estadística de fiabilidad de habilidades sociales

\begin{tabular}{c|c|c}
\hline \multicolumn{3}{|c}{ Estadísticas de fiabilidad } \\
$\begin{array}{c}\text { Alfa de } \\
\text { Cronbach }\end{array}$ & $\begin{array}{c}\text { Alfa de Cronbach basada en } \\
\text { elementos estandarizados }\end{array}$ & N de elementos \\
\hline, 849 &, 850 & 20 \\
\hline
\end{tabular}

La tabla 3 expone el valor de confiabilidad del instrumento, siendo de 0,489 cuya interpretación es excelente. Además, se exponen los 20 ítems.

\subsection{Procedimientos.}

Se ha determinado las diferentes etapas por las que esta investigación atravesó.

- Se ha realizado las coordinaciones previas con la institución educativa Karina Damian Violeta del distrito de Nuevo Chao para la aplicación de la investigación.

- La aplicación del proyecto se realizó por la modalidad virtual en el contexto de la Covid-19 y con el consentimiento informado de los padres de familia de los estudiantes.

- En la etapa del desarrollo de este proyecto se aplicó la ficha del pre y post test a ambos grupos (control y experimental).

- Se aplicó un programa de 15 sesiones. Estas fueron desarrolladas según los procedimientos y dimensiones del programa convivimos en armonía.

- En el grupo control, se desarrolló los temas sobre habilidades sociales con la metodología tradicional.

- Al término, se realizó el análisis de los resultados, discusión y conclusiones. 


\subsection{Método de análisis de datos.}

Las conclusiones de la investigación fueron expresadas a través de la estadística descriptiva por medio del uso de tablas y gráficos estadísticos para la presentación de los resultados, así como también, se hizo uso de los programas Excel y SPPS24.

Asimismo, se utilizó la estadística diferencial para determinar la media aritmética, el coeficiente de variabilidad y la desviación estándar. Para contrastar los datos e identificar si corresponden a una distribución normal se aplicó la prueba de Se utilizó la prueba de normalidad "Shapiro Wilk" $(\mathrm{n}<50)$, con el nivel de significación del 0.05, la misma que arroja un sig < 0,05 en cada uno de los factores utilizados en la investigación, por lo que se rechaza la hipótesis alterna que establece la normalidad de las variables y se acepta la hipótesis nula, por lo que se usará una prueba no paramétrica como es la prueba de Wilcoxon para la contratación de las hipótesis.

\section{RESULTADOS Y DISCUSIÓN}

\subsection{Nivel descriptivo}

\section{Tabla 4}

Niveles de habilidades sociales de los estudiantes en el pre test.

\begin{tabular}{cccccc}
\hline Categorías & Rangos & \multicolumn{2}{c}{ Grupo Experimental } & \multicolumn{2}{c}{ Grupo Control } \\
\cline { 3 - 6 } & & $\mathbf{f}$ & $\mathbf{\%}$ & $\mathbf{f}$ & $\%$ \\
\hline Alto & $28-40$ & 10 & 33,3 & 9 & 30,0 \\
Medio & $15-27$ & 20 & 66,7 & 21 & 70,0 \\
Bajo & $0-14$ & 0 & 0,0 & 0 & 0,0 \\
Total & & $\mathbf{3 0}$ & $\mathbf{1 0 0 , 0}$ & $\mathbf{3 0}$ & $\mathbf{1 0 0 , 0}$ \\
\hline
\end{tabular}

Nota: Data del pre test de la variable dependiente

\section{Interpretación}

En la tabla 4 se puede observar que con relación al grupo experimental en el pre test, que el 33,3\% (10) presentan niveles altos de desarrollo de las habilidades sociales, en un nivel medio se ubica el 66,7\% (20) y ningún estudiante en el nivel bajo; mientras que en el grupo control el 30,0\% (9) se ubican en el nivel alto, el 70\% (21) en el nivel medio y ningún estudiante en el nivel bajo. 


\section{Tabla 5}

Niveles de habilidades sociales de los estudiantes en el pos test

\begin{tabular}{cccccc}
\hline Categorías & Rangos & \multicolumn{2}{c}{ Grupo Experimental } & \multicolumn{2}{c}{ Grupo Control } \\
& & f & \% & f & \% \\
\hline Alto & $28-40$ & 14 & 46,7 & 12 & 40,0 \\
Medio & $15-27$ & 16 & 53,3 & 18 & 60,0 \\
Bajo & $0-14$ & 0 & 0,0 & 0 & 0,0 \\
Total & & $\mathbf{3 0}$ & $\mathbf{1 0 0 , 0}$ & $\mathbf{3 0}$ & $\mathbf{1 0 0 , 0}$ \\
\hline
\end{tabular}

Nota: Data del pos test de la variable dependiente

\section{Interpretación}

En la tabla 5 se puede observar que con relación al grupo experimental en el pos test, que el 46,7\% (14) presentan niveles altos de desarrollo de las habilidades sociales, en un nivel medio se ubica el 53,3\% (16) y ningún estudiante en el nivel bajo; mientras que en el grupo control el 40,0\% (12) se ubican en el nivel alto, el 60\% (21) en el nivel medio y ningún estudiante en el nivel bajo.

\section{Tabla 6}

Nivel de la dimensión Interacción social en los estudiantes según el pre test.

\begin{tabular}{cccccc}
\hline Categorías & Rangos & \multicolumn{2}{c}{ Grupo Experimental } & \multicolumn{2}{c}{ Grupo Control } \\
& & $\mathbf{f}$ & $\mathbf{\text { f }}$ & $\mathbf{\%}$ \\
\hline Alto & $8-10$ & 8 & 26,7 & 7 & 23,3 \\
Medio & $5-7$ & 18 & 60,0 & 21 & 70,0 \\
Bajo & $0-4$ & 4 & 13,3 & 2 & 6,7 \\
Total & & $\mathbf{3 0}$ & $\mathbf{1 0 0 , 0}$ & $\mathbf{3 0}$ & $\mathbf{1 0 0 , 0}$
\end{tabular}

Nota: Data del pre test de la variable dependiente

\section{Interpretación}

En la tabla 6 se puede observar que con relación al grupo experimental en el pre test, que el 26,7\% (8) presentan niveles altos de desarrollo de Interacción social; en un nivel medio se ubica el 60,0\% (18) y ningún estudiante en el nivel bajo; mientras que en el grupo control el 23,3\% (7) se ubican en el nivel alto, el 70\% (21) en el nivel medio y el 6,7\% (2) en el nivel bajo. 


\section{Tabla 7}

Nivel de la dimensión Interacción social en los estudiantes según el pos test

\begin{tabular}{cccccc}
\hline Categorías & Rangos & \multicolumn{2}{c}{ Grupo Experimental } & \multicolumn{2}{c}{ Grupo Control } \\
& & f & f & f & \% \\
\hline Alto & $8-10$ & 12 & 40,0 & 10 & 33,3 \\
Medio & $5-7$ & 18 & 60,0 & 19 & 63,3 \\
Bajo & $0-4$ & 0 & 0,0 & 1 & 3,4 \\
Total & & $\mathbf{3 0}$ & $\mathbf{1 0 0 , 0}$ & $\mathbf{3 0}$ & $\mathbf{1 0 0 , 0}$ \\
\hline
\end{tabular}

Nota: Data del pos test de la variable dependiente

\section{Interpretación}

En la tabla 7 se puede observar que con relación al grupo experimental en el pos test, que el 40,0\% (12) presentan niveles altos de desarrollo de Interacción social; en un nivel medio se ubica el 60,0\% (18) y ningún estudiante en el nivel bajo; mientras que en el grupo control el 33,3\% (10) se ubican en el nivel alto, el 63,3\% (19) en el nivel medio y el $3,4 \%$ (1) en el nivel bajo.

\section{Tabla 8}

Nivel de la dimensión Concepción de amigos en los estudiantes según el pre test

\begin{tabular}{cccccc}
\hline Categorías & Rangos & \multicolumn{2}{c}{ Grupo Experimental } & \multicolumn{2}{c}{ Grupo Control } \\
& & $\mathbf{f}$ & $\mathbf{\text { f }}$ & $\mathbf{\%}$ \\
\hline Alto & $8-10$ & 8 & 26,7 & 8 & 26,7 \\
Medio & $5-7$ & 20 & 66,7 & 19 & 63,3 \\
Bajo & $0-4$ & 2 & 6,6 & 3 & 10,0 \\
Total & & 30 & 100,0 & 30 & 100,0 \\
\hline
\end{tabular}

Nota: Data del pre test de la variable dependiente

\section{Interpretación}

En la tabla 8 se puede observar que con relación al grupo experimental en el pre test, que el 26,7\% (8) presentan niveles altos de desarrollo de la Concepción de amigos; en un nivel medio se ubica el 66,7\% (20) y el 6,6\% (2) en el nivel bajo; mientras que en el grupo control el 26,7\% (8) se ubican en el nivel alto, el 63,3\% (19) en el nivel medio y el 10,0\% (3) en el nivel bajo. 


\section{Tabla 9}

Nivel de la dimensión Concepción de amigos en los estudiantes según el pos test

\begin{tabular}{cccccc}
\hline Categorías & Rangos & \multicolumn{2}{c}{ Grupo Experimental } & \multicolumn{2}{c}{ Grupo Control } \\
& & f & f & \% \\
\hline Alto & $8-10$ & 12 & 40,0 & 10 & 33,3 \\
Medio & $5-7$ & 16 & 53,3 & 18 & 60,0 \\
Bajo & $0-4$ & 2 & 6,7 & 2 & 6,7 \\
Total & & $\mathbf{3 0}$ & $\mathbf{1 0 0 , 0}$ & $\mathbf{3 0}$ & $\mathbf{1 0 0 , 0}$ \\
\hline
\end{tabular}

Nota: Data del pos test de la variable dependiente

\section{Interpretación}

En la tabla 9 se puede observar que con relación al grupo experimental en el pos test, que el 40,0\% (12) presentan niveles altos de desarrollo de la Concepción de amigos; en un nivel medio se ubica el 53,3\% (16) y 6,7\% (2) en el nivel bajo; mientras que en el grupo control el 33,3\% (10) se ubican en el nivel alto, el 60,0\% (18) en el nivel medio y el 6,7\% (2) en el nivel bajo.

\section{Tabla 10}

Nivel de la dimensión Desarrollo de emociones y sentimientos en los estudiantes según el pre test

\begin{tabular}{cccccc} 
Categorías & Rangos & \multicolumn{2}{c}{ Grupo Experimental } & \multicolumn{2}{c}{ Grupo Control } \\
& & f & \% & f & \% \\
\hline Alto & $8-10$ & 7 & 23,3 & 8 & 26,7 \\
Medio & $5-7$ & 19 & 63,3 & 14 & 46,6 \\
Bajo & $0-4$ & 4 & 13,4 & 8 & 26,7 \\
Total & & $\mathbf{3 0}$ & $\mathbf{1 0 0 , 0}$ & $\mathbf{3 0}$ & $\mathbf{1 0 0 , 0}$
\end{tabular}

Nota: Data del pre test de la variable dependiente

\section{Interpretación}

En la tabla 10 se puede observar que con relación al grupo experimental en el pre test, que el 23,3\% (7) presentan niveles altos de desarrollo de emociones y sentimientos; en un nivel medio se ubica el 63,3\% (19) y el 13,4\% (4) en el nivel bajo; mientras que en el grupo control el 26,7\% (8) se ubican en el nivel alto, el 46,6\% (14) en el nivel medio y el $26,7 \%$ (8) en el nivel bajo. 
Culcas Taboada...

\section{Tabla 11}

Nivel de la dimensión Desarrollo de emociones y sentimientos en los estudiantes según el pos test

\begin{tabular}{cccccc}
\hline Categorías & Rangos & \multicolumn{2}{c}{ Grupo Experimental } & \multicolumn{2}{c}{ Grupo Control } \\
& & f & \% & f & \% \\
\hline Alto & $8-10$ & 11 & 36,7 & 8 & 26,7 \\
Medio & $5-7$ & 15 & 50,0 & 18 & 60,0 \\
Bajo & $0-4$ & 4 & 13,3 & 4 & 13,3 \\
Total & & $\mathbf{3 0}$ & $\mathbf{1 0 0 , 0}$ & $\mathbf{3 0}$ & $\mathbf{1 0 0 , 0}$ \\
\hline
\end{tabular}

Nota: Data del pos test de la variable dependiente

\section{Interpretación}

En la tabla 11 se puede observar que con relación al grupo experimental en el pos test, que el 36,7\% (12) presentan niveles altos de desarrollo de emociones y sentimientos; en un nivel medio se ubica el 50,0\% (15) y 13,3\% (4) en el nivel bajo; mientras que en el grupo control el $26,7 \%$ (8) se ubican en el nivel alto, el 60,0\% (18) en el nivel medio y el $13,3 \%$ (4) en el nivel bajo.

\section{Tabla 12}

Nivel de la dimensión Relaciones interpersonales en los estudiantes según el pre test

\begin{tabular}{cccccc}
\hline Categorías & Rangos & \multicolumn{2}{c}{ Grupo Experimental } & \multicolumn{2}{c}{ Grupo Control } \\
& & $\mathbf{f}$ & $\mathbf{\text { f }}$ & $\mathbf{\%}$ \\
\hline Alto & $8-10$ & 7 & 23,3 & 7 & 23,3 \\
Medio & $5-7$ & 14 & 46,7 & 16 & 53,4 \\
Bajo & $0-4$ & 9 & 30,0 & 7 & 23,3 \\
Total & & $\mathbf{3 0}$ & $\mathbf{1 0 0 , 0}$ & $\mathbf{3 0}$ & $\mathbf{1 0 0 , 0}$ \\
\hline
\end{tabular}

Nota: Data del pre test de la variable dependiente

\section{Interpretación}

En la tabla 12 se puede observar que con relación al grupo experimental en el pre test, que el 23,3\% (7) presentan niveles altos de desarrollo de relaciones interpersonales; en un nivel medio se ubica el 46,7\% (14) y el 30,0\% (9) en el nivel bajo; mientras que en el grupo control el 23,3\% (7) se ubican en el nivel alto, el 53,4\% (16) en el nivel medio y el $23,3 \%$ (7) en el nivel bajo. 


\section{Tabla 13}

Nivel de la dimensión Relaciones interpersonales en los estudiantes según el pos test

\begin{tabular}{cccccc}
\hline Categorías & Rangos & \multicolumn{2}{c}{ Grupo Experimental } & \multicolumn{2}{c}{ Grupo Control } \\
& & f & \% & f \\
\hline Alto & $8-10$ & 15 & 50,0 & 9 & 30,0 \\
Medio & $5-7$ & 14 & 46,7 & 16 & 53,4 \\
Bajo & $0-4$ & 1 & 3,3 & 5 & 16,6 \\
Total & & $\mathbf{3 0}$ & $\mathbf{1 0 0 , 0}$ & $\mathbf{3 0}$ & $\mathbf{1 0 0 , 0}$ \\
\hline
\end{tabular}

Nota: Data del pos test de la variable dependiente

\section{Interpretación}

En la tabla 13 se puede observar que con relación al grupo experimental en el pos test, que el 50,0\% (15) presentan niveles altos de desarrollo de Relaciones interpersonales; en un nivel medio se ubica el 53,4\% (16) y $1,3 \%$ (1) en el nivel bajo; mientras que en el grupo control el 30,0\% (9) se ubican en el nivel alto, el 53,4\% (16) en el nivel medio y el $16,6 \%$ (5) en el nivel bajo.

\subsection{Nivel inferencial}

Ho: Las variables de estudio No presentan distribución normal.

Ha: Las variables de estudio presentan distribución normal.

Nivel de significancia: $\alpha=0.05$

Regla de decisión: Si Sig. $=p>\alpha$, se rechaza la hipótesis nula

Si Sig. $=p \leq \alpha$, No se rechaza la hipótesis nula

\section{Tabla 14}

Pruebas de normalidad de Shapiro - Wilk

\begin{tabular}{lcccccc} 
& \multicolumn{3}{c}{ Kolmogorov-Smirnov } & \multicolumn{3}{c}{ Shapiro-Wilk } \\
& Estadístico & gl & Sig. & Estadístico & gl & Sig. \\
\hline Pre test - GE &, 170 & 30 &, 027 &, 922 & 30 &, 029 \\
Pos test - GE &, 199 & 30 &, 004 &, 917 & 30 &, 022 \\
Pre test - GC &, 184 & 30 &, 011 &, 916 & 30 &, 021 \\
Pos test - GC &, 151 & 30 &, 081 &, 929 & 30 &, 046 \\
\hline
\end{tabular}

Nota: a. Corrección de significación de Lilliefors

\section{Interpretación}

La tabla 14, muestran los resultados de la prueba de normalidad "Shapiro Wilk" $(\mathrm{n}<50)$, con el nivel de significación del 0.05, la misma que arroja un sig $<0,05$ en cada uno de 
los factores utilizados en la investigación, por lo que se rechaza la hipótesis alterna que establece la normalidad de las variables y se acepta la hipótesis nula, por lo que se usará una prueba no paramétrica como es la prueba de Wilcoxon para la contratación de las hipótesis.

\section{Contrastación de hipótesis}

\section{Hipótesis general}

$\mathrm{H}_{\mathrm{i}}$ : El programa "convivimos en armonía” influirá significativamente en la mejora de las habilidades sociales en estudiantes de sexto de primaria

$\mathrm{H}_{0}$ : El programa "convivimos en armonía" no influirá significativamente en la mejora de las habilidades sociales en estudiantes de sexto de primaria

\section{Tabla 15}

Prueba de Wilcoxon para las diferencias antes y después de aplicar el programa

\begin{tabular}{lllllll}
\hline \multirow{2}{*}{\multicolumn{1}{c}{ Prueba }} & \multicolumn{3}{c}{ Grupo Experimental } & \multicolumn{3}{c}{ Grupo Control } \\
\cline { 2 - 7 } & $\begin{array}{c}\text { Med Pre } \\
\text { test }\end{array}$ & $\begin{array}{c}\text { Med Pos } \\
\text { test }\end{array}$ & $\begin{array}{c}\text { Med de } \\
\text { dif }\end{array}$ & $\begin{array}{c}\text { Med. } \\
\text { Pre test }\end{array}$ & $\begin{array}{c}\text { Med. Pos } \\
\text { test }\end{array}$ & $\begin{array}{c}\text { Med. } \\
\text { de dif }\end{array}$ \\
\hline Global & 23,00 & 26,00 & 3,00 & 23,00 & 25,00 & 2,00 \\
Prueba Pre - Pos test & $\mathrm{Z}=4,698$ & & $\mathrm{p}<0,01$ & $\mathrm{Z}=4,099$ & $\mathrm{p}<0,01$ \\
Prueba de Med GE- & $\mathrm{Z}=0,599$ & $\mathrm{p}<0,05$ & & & & \\
GC de las dif & & & & & & \\
\hline
\end{tabular}

Nota: $\mathrm{p}<0.05$, es significativo

\section{Interpretación}

Se muestra las diferencias de las medianas entre el pre test y pos test tanto del grupo experimental como del grupo control, las mismas que son significativas; por cuanto el sig. $=0,000<0,05$; por lo que existe evidencia suficiente para rechazar la hipótesis nula afirmando que el programa Vivimos en armonía mejora significativamente el nivel de habilidades sociales de manera global.

\section{Hipótesis especificas}

$\mathrm{H}_{\mathrm{i}}$ : El programa "convivimos en armonía” influirá significativamente en la mejora de la dimensión interacción social en estudiantes de sexto de primaria

$\mathrm{H}_{0}$ : El programa "convivimos en armonía" no influirá significativamente en la mejora de la dimensión interacción social en estudiantes de sexto de primaria 


\section{Tabla 16}

Prueba de Wilcoxon para las medianas antes y después del programa en relación a la dimensión interacción social

\begin{tabular}{lcccccc}
\hline \multirow{2}{*}{ Prueba } & \multicolumn{3}{c}{ Grupo Experimental } & \multicolumn{3}{c}{ Grupo Control } \\
\cline { 2 - 7 } & $\begin{array}{c}\text { Med Pre } \\
\text { test }\end{array}$ & $\begin{array}{c}\text { Med Pos } \\
\text { test }\end{array}$ & $\begin{array}{c}\text { Med de } \\
\text { dif }\end{array}$ & $\begin{array}{c}\text { Med. Pre } \\
\text { test }\end{array}$ & $\begin{array}{c}\text { Med. Pos } \\
\text { test }\end{array}$ & $\begin{array}{c}\text { Med. } \\
\text { De dif }\end{array}$ \\
\hline Global & 6,00 & 7,00 & 1,000 & 6,00 & 6,00 & 0,000 \\
Prueba Pre - Pos & $\mathrm{Z}=3,733$ & $\mathrm{p}<0,01$ & $\mathrm{Z}=2,543$ & $\mathrm{p}<0,05$ \\
test & & & & \\
Prueba de Med & & $\mathrm{Z}=1,190$ & $\mathrm{p}>0,05$ & \\
GE-GC de las dif & & & & & \\
\end{tabular}

Nota: $\mathrm{p}>0.05$, no es significativo

\section{Interpretación}

Se muestra las diferencias de las medianas entre el pre test y pos test tanto del grupo experimental como del grupo control, las mismas que no son significativas; por cuanto el $\mathrm{p}$ valor $=0,0>0,05$; por lo que existe evidencia suficiente para aceptar la hipótesis nula afirmando que el programa Vivimos en armonía no mejora significativamente el nivel de habilidades sociales en su dimensión interacción social.

$\mathrm{H}_{\mathrm{i}}$ : El programa "convivimos en armonía" influirá significativamente en la mejora de la dimensión concepción de amigos en estudiantes de sexto de primaria

$\mathrm{H}_{0}$ : El programa "convivimos en armonía" no influirá significativamente en la mejora de la dimensión concepción de amigos en estudiantes de sexto de primaria

\section{Tabla 17}

Prueba de Wilcoxon para las medianas antes y después del programa en relación a la dimensión concepción de amigos.

\begin{tabular}{lcccccc}
\hline \multirow{2}{*}{ Prueba } & \multicolumn{3}{c}{ Grupo Experimental } & \multicolumn{3}{c}{ Grupo Control } \\
\cline { 2 - 7 } & $\begin{array}{c}\text { Med Pre } \\
\text { test }\end{array}$ & $\begin{array}{c}\text { Med Pos } \\
\text { test }\end{array}$ & $\begin{array}{c}\text { Med } \\
\text { de dif }\end{array}$ & $\begin{array}{c}\text { Med. Pre } \\
\text { test }\end{array}$ & $\begin{array}{c}\text { Med. Pos } \\
\text { test }\end{array}$ & $\begin{array}{c}\text { Med. } \\
\text { De dif }\end{array}$ \\
\hline Global & 6,00 & 7,00 & 1,000 & 6,00 & 7,00 & 0,000 \\
Prueba Pre - Pos & $\mathrm{Z}=2,386$ & $\mathrm{p}<0,05$ & $\mathrm{Z}=2,980$ & $\mathrm{p}<0,05$ \\
test & & & & \\
Prueba de Med & & $\mathrm{Z}=0,364$ & $\mathrm{p}>0,05$ & \\
GE-GC de las dif & & & & & \\
\hline
\end{tabular}

Nota: $\mathrm{p}>0.05$, no es significativo 


\section{Interpretación}

Se muestra las diferencias de las medianas entre el pre test y pos test tanto del grupo experimental como del grupo control, las mismas que no son significativas; por cuanto el $\mathrm{p}$ valor $=0,0>0,05$; por lo que existe evidencia suficiente para aceptar la hipótesis nula afirmando que el programa Vivimos en armonía no mejora significativamente el nivel de habilidades sociales en su dimensión interacción social.

$\mathrm{H}_{\mathrm{i}}$ : El programa “convivimos en armonía” influirá significativamente en la mejora de la dimensión desarrollo de emociones y sentimientos en estudiantes de sexto de primaria $\mathrm{H}_{0}$ : El programa “convivimos en armonía” no influirá significativamente en la mejora de la dimensión desarrollo de emociones y sentimientos en estudiantes de sexto de primaria

\section{Tabla 18}

Prueba de Wilcoxon para las medianas antes y después del programa en relación a la dimensión Desarrollo de emociones y sentimientos.

\begin{tabular}{lcccccc}
\hline \multirow{2}{*}{ Prueba } & \multicolumn{3}{c}{ Grupo Experimental } & \multicolumn{3}{c}{ Grupo Control } \\
\cline { 2 - 7 } & $\begin{array}{c}\text { Med Pre } \\
\text { test }\end{array}$ & $\begin{array}{c}\text { Med Pos } \\
\text { test }\end{array}$ & $\begin{array}{c}\text { Med de } \\
\text { dif }\end{array}$ & $\begin{array}{c}\text { Med. } \\
\text { Pre test }\end{array}$ & $\begin{array}{c}\text { Med. Pos } \\
\text { test }\end{array}$ & $\begin{array}{c}\text { Med. De } \\
\text { dif }\end{array}$ \\
\hline Global & 6,00 & 6,50 & 1,000 & 5,50 & 6,00 & 0,000 \\
Prueba & Pre-Pos & $\mathrm{Z}=1,999$ & $\mathrm{p}<0,05$ & $\mathrm{Z}=2,493$ & $\mathrm{p}<0,05$ \\
test & & & & & \\
Prueba de Med & & $\mathrm{Z}=0,477$ & $\mathrm{p}>0,05$ & \\
GE-GC de las dif & & & & & \\
\hline
\end{tabular}

Nota: $\mathrm{p}>0.05$, no es significativo

\section{Interpretación}

Se muestra las diferencias de las medianas entre el pre test y pos test tanto del grupo experimental como del grupo control, las mismas que no son significativas; por cuanto el $\mathrm{p}$ valor $=0,0>0,05$; por lo que existe evidencia suficiente para aceptar la hipótesis nula afirmando que el programa Vivimos en armonía no mejora significativamente el nivel de habilidades sociales en su dimensión desarrollo de emociones y sentimientos.

$\mathrm{H}_{\mathrm{i}}$ : El programa "convivimos en armonía” influirá significativamente en la mejora de la dimensión relaciones interpersonales en estudiantes de sexto de primaria $\mathrm{H}_{0}$ : El programa “convivimos en armonía" no influirá significativamente en la mejora de la dimensión relaciones interpersonales en estudiantes de sexto de primaria 


\section{Tabla 19}

Prueba de Wilcoxon para las medianas antes y después del programa en relación a la dimensión relaciones interpersonales.

\begin{tabular}{lcccccc}
\hline \multirow{2}{*}{ Prueba } & \multicolumn{3}{c}{ Grupo Experimental } & \multicolumn{3}{c}{ Grupo Control } \\
\cline { 2 - 7 } & $\begin{array}{c}\text { Med Pre } \\
\text { test }\end{array}$ & $\begin{array}{c}\text { Med Pos } \\
\text { test }\end{array}$ & $\begin{array}{c}\text { Med de } \\
\text { dif }\end{array}$ & $\begin{array}{c}\text { Med. Pre } \\
\text { test }\end{array}$ & $\begin{array}{c}\text { Med. Pos } \\
\text { test }\end{array}$ & $\begin{array}{c}\text { Med. } \\
\text { De dif }\end{array}$ \\
\hline Global & 6,00 & 7,00 & 1,000 & 6,00 & 6,00 & 0,000 \\
Prueba Pre - Pos & $\mathrm{Z}=3,906$ & $\mathrm{p}<0,01$ & $\mathrm{Z}=2,725$ & $\mathrm{p}<0,01$ \\
test & & & & \\
Prueba de Med & & $\mathrm{Z}=2,525$ & $\mathrm{p}>0,05$ & \\
GE-GC de las dif & & & & \\
\hline
\end{tabular}

Nota: $\mathrm{p}>0.05$, no es significativo

\section{Interpretación}

Se muestra las diferencias de las medianas entre el pre test y pos test tanto del grupo experimental como del grupo control, las mismas que no son significativas; por cuanto el $\mathrm{p}$ valor $=0,0>0,05$; por lo que existe evidencia suficiente para aceptar la hipótesis nula afirmando que el programa Vivimos en armonía no mejora significativamente el nivel de habilidades sociales en su dimensión relaciones interpersonales.

\section{CONCLUSIÓN O CONSIDERACIONES FINALES}

\subsection{Discusión}

Después de procesar los resultados y hacer el análisis estadístico respectivo, se procede a realizar la discusión de los mismos mediante la técnica de la triangulación.

Así se tiene que, en relación al objetivo general, la prueba de Wilcoxon tal como se muestra en la tabla 12, evidencia una diferencia significativa en las medianas del grupo control y grupo experimental, considerando los resultados del pre tes y pos test. De la cual se infiere que el programa en términos globales, ha influenciado significativamente en la mejora de las habilidades sociales de los estudiantes de sexto grado.

Estos hallazgos concuerdan totalmente con los aportes de Pullchs, (2018). Y Casallo, (2018). Quien después de realizar su estudio, sostenían que la aplicación del programa de habilidades sociales logra resultados significativos en el desarrollo de las habilidades sociales iniciales: como por ejemplo dar las gracias, formular preguntas, saber escuchar, mantener una conversación e iniciar, hacer cumplidos a las personas y presentarse. También ayuda a que los niños adquieran habilidades sociales para vivir en un ambiente 
armónico, ayuda a reconocer sentimientos y a expresarlos de una manera adecuada frente a los demás. El programa ayudó que los alumnos desarrollen y apliquen de manera eficaz diferentes habilidades sociales dándose cuenta de cuándo y cómo utilizar habilidades sociales para con sus semejantes.

Por otro lado, se concuerda parcialmente con los aportes de Rubiales, et al. (2018). Por cuanto argumentaban que los programas de entrenamiento Socioemocional para niños y adolescentes de 6 a 18 años, permiten que los estudiantes logren relajarse, tener regulación de emociones, ser asertivos, desarrollen su salud mental, y logren una buena relación con los demás, además estos programas son beneficiosos porque los niños y adolescentes generaron una adecuada comunicación con los demás, siendo pertinentes cuando se les presenta un conflicto en la escuela, o en casa. Sin embargo, cuando estos se realizan a distancia usando los entornos virtuales, no se logra los objetivos propuestos.

En esa misma línea de ideas, (Vigotsky, 1997) sostiene que el ser humano es producto de un desarrollo histórico social donde el lenguaje juega un rol esencial como herramienta para confortar relaciones interpersonales, dialogar y generar actitudes personales positivas. Así mismo (Bandura, 1977). Lo refuerza desde su teoría del aprendizaje social que: que al hablar de habilidades sociales se hace referencia a conductas aprendidas, esto se puede predecir, describir y precisar analizando los diferentes principios de la teoría de aprendizaje social. Para (Amay, 2017) las habilidades sociales se adquieren y aprenden durante el largo de nuestra vida, relacionándose con una variedad de patrones de comportamientos puestos en acción por los seres humanos dentro de la familia y escuela, generando una modificación en el aprendizaje. Todos estos aportes son considerados en entornos presenciales del ser humano.

Desde esta perspectiva se afirma entonces que las habilidades sociales son aprendidas en el entorno social donde se desenvuelve la persona, así mismo al combinarlo con el comportamiento va permitir desarrollar acciones positivas o negativas según lo que adquirió durante el proceso de empoderamiento de estas. También cabe recalcar que los autores hablan sobre la capacidad de inferir, analizar, percibir las conductas que poseen las demás personas. Vigotsky y Bandura hablan de un proceso histórico social por el que pasa las personas para poder comunicarse y por ende comportase. Las personas deben combinar el talento humano con sus habilidades, destrezas y conocimientos para solucionar problemas que se le presentan en la vida cotidiana. 
En relación al primer objetivo específico, la prueba de Wilcoxon tal como se muestra en la tabla 13, evidencia una diferencia no significativa en las medianas del grupo control y grupo experimental, considerando los resultados del pre tes y pos test. De la cual se infiere que el programa no ha influenciado significativamente en la mejora de las interacciones sociales de los estudiantes de sexto grado.

A partir de estos hallazgos se encuentran divergencias con los aportes de (RamírezCoronel et.al., 2020). Cuando argumenta que Las habilidades sociales están en constante cambio debido al contexto donde se desarrolla la persona, son comportamientos que se observan, que se adquieren y que tienen fines sólidos, sin embargo, esto no es posible cuando los estudiantes interactúan en entornos virtuales, sobre todo en las zonas rurales. Por otro lado, León y Lacunza (2020). Expresaban a la luz de sus resultados que para tener amigos es indispensable desarrollar habilidades sociales de manera sistemática y graduada esto permitirá que cuando se encuentren pasando por algún conflicto puedan salir victoriosos de ellas. Los autores consideran que es importante trabajar las habilidades sociales con niños de educación primaria, esto ayudara a ganar más amigos, y fortalecer las relaciones interpersonales, además se deben involucrar otras habilidades, lo que se complementaran.

En esa misma línea de ideas y considerando el contexto de la virtualidad en el que se desarrolló el programa, las divergencias entorno a los aportes de (González, 2017) son amplias, puesto que sostenía que los estudiantes al trabajar con la TICS se muestran más conscientes de sus emociones, al trabajar con autonomía se desarrollan plácidamente en el desarrollo de la inteligencia interpersonal e intrapersonal, desarrollan más curiosidad por los problemas de la vida al estar motivados, esto conllevara a lograr y cumplir todo lo que anhelan y expresar como se sienten. Otro factor para el desarrollo de habilidades sociales es el adecuado uso de las TICS, esto permitirá que el niño interactúe con sus compañeros regulando y expresando sus emociones hacia los demás, respetando los turnos al hablar y generando competencias digitales.

Así mismo desde la perspectiva teórica de (Vigotsky, 1997) se sostiene que el ser humano es producto de un desarrollo histórico social donde el lenguaje juega un rol esencial como herramienta para confortar relaciones interpersonales, dialogar y generar actitudes personales positivas. Así mismo (Bandura, 1977). Lo refuerza desde su teoría del aprendizaje social que: que al hablar de habilidades sociales se hace referencia a 
conductas aprendidas, esto se puede predecir, describir y precisar analizando los diferentes principios de la teoría de aprendizaje social.

En relación al segundo objetivo específico, los resultados que derivan de la prueba de Wilcoxon tal como se muestra en la tabla 13, evidencia una diferencia no significativa en las medianas del grupo control y grupo experimental, considerando los resultados antes y después de la aplicación del programa. De la cual se infiere que el programa no ha influenciado significativamente en la mejora de la concepción de amigos que tienen los estudiantes de sexto grado, como parte del desarrollo de sus habilidades sociales.

Estos hallazgos distan mucho de los resultados que se plantean en los trabajos de investigación de (Corrales, et al., 2017). Quienes sostienen en respaldo de sus resultados que las habilidades sociales sirven para poder actuar en dificultades de agresión en pares, utilizando una oportuna comunicación asertiva, donde el estudiante logre ser empático con los demás reflexionando sobre los conflictos que se presenta y proponiendo alternativas de solución. Las habilidades sociales permitirán que la relación entre pares sea más armónica, además nos dará como resultado desarrollo de nuevas habilidades en los niños.

Sin embargo, se concuerda parcialmente con los aportes de (Horna-Clavo, et al., 2020) quienes en su artículo científico: Relación de habilidades sociales y tipos de familia en preescolares argumentaban en base a sus resultados que gran parte de los estudiantes de educación básica tiene un nivel de habilidad social bajo, esto depende de la relación y de cómo está conformada su familia, los niños con familias extendidas desarrollan mejor sus habilidades sociales a diferencia de los hogares nucleares, además concluye que es de suma urgencia crear políticas educativas donde se pueda acceder a servicios psicológicos y de psicopedagogía con el único objetivo de mejorar las relaciones sociales entre los estudiantes y disminuir comportamientos y actitudes violentes en la escuela y familia.

En toda institución educativa se debe desarrollar las relaciones interpersonales entre los miembros de la comunidad educativa esto ayudará al adecuado desenvolvimiento y desarrollo de las tareas que se les asigne, esto conllevará a una buena integración y funcionamiento entre todos. Una de las habilidades sociales que se debe desarrollar en la escuela es la adecuada relación de los agentes educativos esto permitirá ayudar a desenvolverse, al desarrollo de tareas y a la integración, generando una comunicación armoniosa. 
Desde la teoría sociocultural se reafirma entonces, que las habilidades sociales son aprendidas en el entorno social donde se desenvuelve la persona, así mismo al combinarlo con el comportamiento va permitir desarrollar acciones positivas o negativas según lo que adquirió durante el proceso de empoderamiento de estas.

En relación al tercer objetivo específico, los resultados que derivan de la prueba de Wilcoxon tal como se muestra en la tabla 14, evidencia una diferencia no significativa en las medianas del grupo control y grupo experimental, considerando los resultados antes y después de la aplicación del programa. De la cual se infiere que el programa no ha influenciado significativamente en la mejora del desarrollo de emociones y sentimientos que tienen los estudiantes de sexto grado, como parte de la consolidación de sus habilidades sociales.

Estos resultados encuentran amplias divergencias con los aportes de (Canet-Juric, et al., 2020). Quienes sostienen en respaldo de sus resultados que el programa AR logró mejorar habilidades sociales, emocionales y cognitivas, las cuales son esenciales para el desarrollo de edad temprana y edad escolar. Por otro lado, también permite tener un rendimiento académico favorable, el desarrollo de este programa logra deslindar cualquier patrón negativo en los niños, como agresiones mala comunicación entre pares falta de empatía y comportamientos negativos, este programa aporta la adquisición de habilidades sociales favorables para su interrelación.

Sin embargo, debido al contexto de virtualidad en el que se ha desarrollado el programa, se concuerda totalmente con los aportes de (Downey \& Gibbs, 2020). Quienes sostienen que los niños con mucha exposición a las pantallas exhiben trayectorias de habilidades sociales similares en comparación con los niños con poca exposición a las pantallas. Hay una excepción notable: las habilidades sociales son menores para los niños que acceden a los juegos en línea y las redes sociales muchas veces al día. Sin embargo, en general, los resultados representan un desafío para la narrativa dominante de que las habilidades sociales están disminuyendo debido al cambio tecnológico. Las habilidades sociales debido a la coyuntura que estamos pasando están siendo opacadas por la tecnología debido que los estudiantes no están interactuando de manera personal con sus pares, no permiten desarrollar habilidades sociales, los maestros al evaluar dichas habilidades no pueden dar un análisis adecuado de los niños ya que este se encuentra detrás de un monitor. 
Finalmente, los resultados de (Buenabad, et al., 2020). No permiten asumir una concordancia total o parcial de ideas; por cuanto argumentan que, la estimulación de los padres y maestros será de suma relevancia en el grupo, esto permitirá un mejor resultado en el desarrollo de habilidades sociales. En esa misma línea de ideas (López \& López, 2018): consideraban que gestionar habilidades sociales en los estudiantes permitirá tener un rendimiento académico optimo, además la familia y el docente cumple un rol fundamental como guía y refuerzo, ellos seleccionaran conductas oportunas cuando se relacionen con los demás. Situaciones que se ven limitadas en los entornos virtuales.

En esta investigación los autores describen el desempeño académico, y la relación con las habilidades sociales que se adquieren en un grupo permitirá que el estudiante identifique emociones propias y de las personas que lo rodean, además que valoren la importancia de la presencia de las habilidades sociales dentro del aula mejorando de forma significativa el desempeño académico esperado en el estudiante, esta investigación también logra reconocer factores culturales y sociales en el cual se desarrollen cada estudiante, el docente también tiene un rol fundamental y necesario dentro de las aulas debe contribuir como guía acompañaste en las diferentes capacidades en el perfil de egreso que se espera de cada estudiante, analizando, conociendo y valorando los diversos desarrollos afectivos de cada etapa.

Desde la perspectiva teórica de (Monjas, 2007) las habilidades sociales son diferentes habilidades y capacidades que el ser humano realiza complementándolas para desarrollar tareas de índole interpersonal y social generando relaciones satisfactorias; siendo estas aprendidas en el entorno social donde se desenvuelve la persona, así mismo al combinarlo con el comportamiento va permitir desarrollar acciones positivas o negativas según su proceso de empoderamiento. En tal sentido Vygotsky y Bandura hablan de un proceso histórico social por el que pasa las personas para poder comunicarse y por ende comportase.

En relación al cuarto objetivo específico, los resultados que derivan de la prueba de Wilcoxon tal como se muestra en la tabla 15 , evidencia una diferencia no significativa en las medianas del grupo control y grupo experimental, considerando los resultados antes y después de la aplicación del programa. De la cual se infiere que el programa no ha influenciado significativamente en la mejora de las relaciones interpersonales en los estudiantes de sexto grado, como parte del desarrollo de sus habilidades sociales. 
A la luz de los resultados no es posible establecer puntos de concordancia con los aportes de (Zhang, et al., 2019), quienes sostienen que el uso de los entornos virtuales permitió no solamente adquirir habilidades sociales si no también el desarrollo de alfabetización digital, pensamiento creativo y motivas más a los alumnos a trabajar por estos medios. Afirmando además que para una óptima adquisición de habilidades académicas los nidos de pequeños tienen que adquirir habilidades cognitivas habilidades sociales y regulación de comportamiento, esto conllevara que los niños generen habilidades sociales óptimos en las escuelas, desarrollando una comunicación asertiva y empática en un contexto en la cual se incluye el uso de la tecnología.

Según Vásquez, (2018). realizo la siguiente investigación: Efecto de un programa de tutoría socio afectiva en la disminución de la conducta antisocial de los estudiantes de sexto grado de primaria de, Lima. La investigación llegó a la siguiente conclusión: las estrategias de técnicas de dialogo, técnicas del modelado, trabajo en equipo, juego de roles desarrollan habilidades sociales en los niños estas habilidades fortalecen las relaciones interpersonales. Al desarrollar la investigación se observa conductas antisociales donde los estudiantes no respetan las normas de convivencia generando actitudes negativas entre los niños a tal grado de sobrepasar las órdenes del docente en este programa se desarrollaron diferentes sesiones donde está presente el área de tutoría: autoestima, la familia la asertividad la empatía la importancia del buen trato.

Sin embargo, es posible concordar con los aportes de (Herrera, et al. (2017). Cuando sostiene que las relaciones interpersonales que se generan en la escuela son estrechamente direccionadas con el rendimiento académico, social y personal, así los estudiantes adquieran más amigos desde muy pequeños, esto genera tener una buena relación social, desplazando conductas de agresión y exclusión. Menciona también que en las escuelas se debe desarrollar habilidades como la responsabilidad social empatía y relaciones interpersonales dentro de las aulas. Concluye también que el aprendizaje emocional y social se debe incluir como parte de estudio en la zona rural.

Por otro lado, si bien es cierto que el programa se ha desarrollado en un contexto de virtualidad, no es posible establecer concordancia alguna con (González, 2017) cuando sostiene que los estudiantes al trabajar con la TICS se muestran más conscientes de sus emociones, al trabajar con autonomía se desarrollan plácidamente en el desarrollo de la inteligencia interpersonal e intrapersonal, desarrollan más curiosidad por los problemas 
de la vida al estar motivados, esto conllevara a lograr y cumplir todo lo que anhelan y expresar como se sienten. En ese sentido otro factor para el desarrollo de habilidades sociales es el adecuado uso de las TICS, esto permitirá que el niño interactúe con sus compañeros regulando y expresando sus emociones hacia los demás, respetando los turnos al hablar y generando competencias digitales.

En esa misma línea de ideas se concuerda totalmente con (Downey \& Gibbs, 2020). Cuando afirman a partir de su investigación, que los niños con mucha exposición a las pantallas exhiben trayectorias de habilidades sociales similares en comparación con los niños con poca exposición. En general, las habilidades sociales están disminuyendo debido al cambio tecnológico. Las habilidades sociales debido a la coyuntura que estamos pasando están siendo opacadas por la tecnología debido que los estudiantes no están interactuando de manera personal con sus pares, no permiten desarrollar habilidades sociales, los maestros al evaluar dichas habilidades no pueden dar un análisis adecuado de los niños ya que este se encuentra detrás de un monitor.

En ese contexto Vigotsky y Bandura hablan de un proceso histórico social por el que pasa las personas para poder comunicarse y por ende comportase. Para (Monjas, 2007) Las habilidades sociales con diferentes habilidades y capacidades que el ser humano realiza complementándolas para desarrollar tareas de índole interpersonal y social generando relaciones satisfactorias.

\section{LISTA DE REFERENCIAS}

Almaraz,D.,Coeto,G,.\& Camacho,E.,(2019). Habilidades Sociales En Niños De Primaria Universidad Autónoma del Estado de México, México. Red de Investigadores Educativos ChihuahuaA.C.10(19),191206.DOI:http://dx.doi.org/10.33010/ie_rie_rediech.v10i19.706

Amay, A. (2017). Habilidades Sociales. Editorial THGY. https://bit.ly/2RE0wVM Andueza. (2015). Educar las relaciones interpersonales . España. https://bit.ly/3qqxP14 Arrigoni, F., \& Solans, A. (2018). PROGRAMA DE PROMOCIÓN DE HABILIDADES SOCIALES (PHAS). RUEDES, 65 -85. https://bit.ly/3bU93hs

Astochado, D. y. (2014). Habilidades Sociales. Lima: https://bit.ly/3ce6xlr.

Bandura. (1977). Teoria del aprendizaje social. New Jersey: Englewood. Revista Latinoamericana de Psicología,41(1),109-119. https://bit.ly/3oiYJp7 
Bisquerra. (2003). Relaciones Interpersonales. Mexico: Editorial Mac Graw Hill. https://repositorio.unprg.edu.pe/handle/20.500.12893/8638

Buenabad, N., Sánchez, R. ,., Gutiérrez, M., Díaz, A., Ortiz, A., González, T., . . . Villatoro, J. (2020). Cluster Randomized Trial of a Multicomponent SchoolBased Program in Mexico to Prevent Behavioral Problems and Develop Social Skills in Children. Child \& Youth Care Forum; 49, 343-364.DOI: 10.1007/s10566-019-09535-3.

Caballo. (2005). Evaluación de las habilidades sociales. Madrid: Pirámide. https://bit.ly/3Hbv6hO

Caballo. (2006). Manual de evaluación y entrenamiento de las habilidades sociales. España: Editores Siglo XXI. https://bit.ly/308FMNg

Caballo, C. O. (2015). Manual de conductual. España. Manual de psicología.https://bit.ly/30kFHX8

Cabello Cuenca, E., Pérez Escoda, N., Ros Morente, A., \& Filella Guiu, G. (2019). Los programas de educación emocional happy 8-12 and happy 12-16. Evaluación de su impacto en las emociones y el bienestar. REOP - Revista Española De Orientación $\quad$ Y $\quad$ Psicopedagogía, 30(2), 53-66. https://doi.org/10.5944/reop.vol.30.num.2.2019.25338

Calderón, D. L. (2015). la relación entre el clima social familiar y las habilidades sociales. piramide. https://bit.ly/3wzCVJC

Canet-Juric, L., García-Coni, A., Andrés, M. L., Vernucci, S., Aydmune, Y.; Stelzer, F. \& Richard's, M. M. (2020). Intervención sobre autorregulación cognitiva, conductual y emocional en niños: Una revisión de enfoques basados en procesos y en el currículo escolar, en Argentina. Revista Argentina de Ciencias del Comportamiento, 12(1), 1-25. https://doi.org/10.32348/1852.4206.v12.n1.24999

Carrillo. (2015). Validadción de un programa lúdico para la mejora de las habilidades sociales en niños de 9 a 12 años. [Tesis doctoral,dissertation]. Universidad de España. https://bit.ly/3kk4amb

Casallo, L. (2018). Desarrollo de las habilidades sociales para reducir conductas agresivas. Junín: y [Tesis doctoral,dissertation].,UNIVERSIDAD NACIONAL DE EDUCACIÓN.https://bit.ly/2RHKLgu. 
Castro. (2011). Las relaciones interpersonales en la transición de los estudiantes. Revista Electrónica Educare, 193-210. https://bit.ly/3bVw43F

Castro, G. (2013). La visión del profesorado de Educación Infantil y Primaria de Cantabria sobre la participación y las relaciones interpersonales entre la comunidad escolar . Aula Abierta , 73-84. https://bit.ly/309fMSv

Castro, M., \& Ramírez, R. (2019). Aplicación de la psicomotricidad como herramienta de inclusión social en educación infantil. ESHPA - Education, Sport, Health and Physical Activity;3(2)., 23-234. https://bit.ly/30g5DTV

Chávez, M., \& Aragón, L. (2017). Habilidades sociales y conductas de bullying. Digital Internacional de Psicología y Ciencia Social; 3(1), 21-37. https://bit.ly/30g5DTV

- Combs, S. (2007). Programa de desarrollo de habilidades sociales. Santiago: Universitaria. https://bit.ly/3F3cw9O

Corrales , A., Quijano, N,. \& Góngora, Elías.(2017). Empatía, Comunicación Asertiva Y Seguimiento De Normas. Un Programa Para Desarrollar Habilidades Para La Vida. Enseñanza e Investigación en Psicología,22(1),58-65. https://bit.ly/3H6hvsc

Dagal, A. (2017). Investigation of the Relationship between Communication Skills, Social Competence and Emotion Regulation Skills of Preschool Children in Turkey. Educational Research and Reviews;12 (4), 164-171,DOI: 10.5897/ERR2017.3139.

Diaz, H. (2001). Estrategias docentes para un aprendizaje significativo. Mexico: McGraw-HII. https://bit.ly/3korBLo

Diaz, M. (2017). Programa de estrategias metodológicas participativas para mejorar habilidades sociales en los estudiantes de la ie no 16005 "pedro paulet mostajo"de jaén-2016. Universidad Cesar Vallejo, Chiclayo. Chiclayo: Tesis doctoral dissertation, Universidad Cesar Vallejo,https://bit.ly/2H6SRx1.

Dominguez, R.(2019). Programa de habilidades sociales en las conductas agresivas de estudiantes de primaria, 2019. [Doctoral dissertation, Universidad Cesar Vallejo]. https://bit.ly/3GYrTIV

Downey, D., \& Gibbs, B. (2020). Kids These Days: Are Face-to-Face Social Skills among American Children Declining?.University of Chicago Press, 125(4), 10301083.https://bit.ly/2RE0wVM 
Fernandez, C. (2001). La educacion del ser emocional. Santiago de Chile: Cuarto propio.Índigo. https://bit.ly/31ROX5P

Fernández, L. (2019). Habilidades sociales en la practica docente: una mirada desde los actores de la educacion básica. Revista Electrónica Científica de Investigación Educativa, 4(2), 1303-1315,https://bit.ly/2FNSS8y.

GONZALEZ, A. R. (2004). METODOLOGIA DE LA INVESTIGACION CIENTIFICA. COLOMBIA: Pontifica Universidad Javeriana. https://bit.ly/3bVN5un

González,R.(2017).Desarrollo de la inteligencia interpersonal e intrapersonal en educación primaria a partir del uso de tecnologías de información y comunicación: estudio de casos. Educación y Tecnología desde una visión Transformadora, 112. https://bit.ly/3EYCcEA

Gutiérrez. (2015). Autoconcepto, dificultades interpersonales, habilidades sociales y conductas asertivas. ISSN electrónico, 42 - 58.

Gutierrez, P. (2015). Autoconcepto, dificultades interpersonales, habilidades sociales y conductas asertivas en adolescentes. España. https://dialnet.unirioja.es/servlet/articulo?codigo=5224079

Herrera, L., Buitrago, R. E., Cepero, S. (2017). Emotional Intelligence in Colombian Primary School Children. Location and Gender Analysis. Universitas Psychologica, 16(3), xx-xx. https://doi.org/10.11144/Javeriana.upsy16-3.eips

Horna-Clavo,E; Arhuis-Inca,W; Bazalar-Palacios,J(2020) en su artículo científico: Relación de habilidades sociales y tipos de familia en preescolares: estudio de caso Revista Virtual. Fundación Universitaria Católica del Norte Colombia,6,224232.DOI: https://doi.org/10.35575/rvucn.n61a13

Jaramillo, B., \& Guzmán, N. (2019). Las Habilidades Sociales en Ambientes Escolares. Universidad Católica Luis Amigó;(3), 151-162. DOI: https://doi.org/10.21501/25907565.3263

Jeffrey. (1982). Evaluación y entrenamiento de las habilidades sociales define a las habilidades sociales. Madrid: Pirámide. https://bit.ly/3qtRiOB

León, G.,Lacunza, A.(2020).Autoestima y habilidades sociales en niños del Gran San Miguel de Tucumán; Ministerio de Salud de Argentina.Revista Argentina de Salud Pública; 11; 42; 3-2020; 22-31. https://bit.ly/30bhsKR 
Linehan. (1984). Efectividad Interpersonal En Situaciones Asertivas. Nueva York: Guilfor Press. https://bit.ly/3n0xAYr

Liwensohn, L. y. (1999). El concepto de habilidad social con especial referencia al comportamiento de las personas deprimidas. Revista de Consultoría y Psicología Clínica, 40, 304-312. https://bit.ly/3F3cw9O

Lopez,L., \& Lopez,B.(2018): El desarrollo de las habilidades socioemocionales como factor influyente en el desempeño académico.UNID sede Tejupilco, México.Revista Atlante: Cuadernos de Educación y Desarrollo (agosto 2018). https://bit.ly/3C2nwTf.

Lorente. (2014). Efecto de la competencia social, la empatía y la conducta prosocial en adolescentes. Valencia . https://bit.ly/3DhxzVX

Mata, S., Gómez, M., \& Dolores, M. (2018). Resolución de problemas interpersonales en niños en exclusión social: Valoración de un programa de entrenamiento. Revista Latinoamericana;50(2), 107-116.

Mendoza, B., \& Maldonado, V. (2017). Acoso escolar y habilidades sociales en alumnado de educación básica. C I E N C I A e r g o -s um; 24(2), 109-116.

Milicic, A. (2011). Climas sociales toxicoa y climas sociales nutritivos para el desarrollo personal en el contexto escolar. . Psykhe, 9. https://bit.ly/3EZ4ylu

Monjas, I. (2007). Cómo promover la convivencia: Programa de Asertividad y Habilidades Sociales (PAHS). Madrid: CEPE. Madrid. CEPE. Madrid: CEPE. https://bit.ly/3FhNcNJ

Mooij, B., Fekkes, M., Scholte, R., \& Overbeek, G. (2020). Effective Components of Social Skills Training Programs for Children and Adolescents in Nonclinical Samples: A Multilevel Meta-analysis. Clinical Child and Family Psychology Review; 23, 250-264,doi: 10.1007/s10567-019-00308-x.

Morales, F. (2017). Relaciones entre afrontamiento del estrés cotidiano,autoconcepto, habilidades sociales e inteligenciaemocional. European Journal of Education and Psychology;10, 41---48.

Navas. (1991). Cómo controlar su ansiedad en situaciones de evaluación o examen. Santo Domingo: CPE. https://bit.ly/3F363f7

Oliveira,J. Bezerra,T. Souza,P. Ataida,M. Santos,F. \& Santos, A.( 2021).Treinamento em Habilidades Sociais na Pré-escola: uma Revisão de Intervenções Brasileiras. 
Instituto Superior Politécnico Gaya Psicologia, Educação e Cultura. Brazil .5(1).https://bit.ly/3EMUqsH.

Pena Garrido, M., Extremera Pacheco, N., \& Rey Peña, L. (2017). Las competencias emocionales: material escolar indispensable en la mochila de la vida. Padres Y Maestros / Journal of Parents and Teachers, (368), 6-10. https://doi.org/10.14422/pym.i368.y2016.001

Peñafield. (2010). Habilidades sociales una herramienta para el éxito. España: Editorial Martínez Roca. https://bit.ly/3H7UZPS

Phillips. (1978). The social skills basis of psychopathology. Nueva York: Grune and Stratton. https://bit.ly/2YwHCHk

Porto,M.,Puerta-Morales,L.,Gelves-Ospina,M.,Urrego-Betancourt,Y. (2021). Funciones Ejecutivas y Rendimiento Académico en Educación Primaria de la Costa Colombiana. Electronic Journal of Research in Educational Psychology,19(2), 351-368. https://bit.ly/3kbsCXc.

Pullchs, M. (2018). Programa educativo para la mejora de las habilidades sociales en estudiantes adolescentes. Arequipa: Tesis doctoral, dissertation,UNIVERSIDAD NACIONAL DE SAN AGUSTÍN DE AREQUIPA,https://bit.ly/2ZVuRnk.

Ramírez-Coronel AA., Martínez-Suárez PC, Cabrera-Mejía JB, Buestán-Andrade PA, Torracchi-Carrasco E, Carpio MG.(2020).Habilidades sociales y agresividad en la infancia y adolescencia. Revista AVFT Archivos Venezolanos de Farmacología y Terapéutica, Volumen 39, número 2,https://bit.ly/3n2mq5E

Rebollo, E., \& de la Peña, ,. (2017). Estudio de la Inteligencia Emocional y Función Ejecutiva en Educación. REIDOCREA;6(3), 29-36.

Reyes, M. (2017). Efectos del taller de estrategias comunicativas en las habilidades sociales. Lima: Tesis Docgtoral dissertation,Universidad Cesar Vallejo,https://bit.ly/2RINHcM.

Robert, M. (1978). "Habilidades sociales necesarias en la adolescencia". España: Pirámide. https://bit.ly/3H4vHSy

Rodriguez, C. A. (2017). Habilidades de argumentación. Una propuesta para el planteamiento de posibles soluciones a los conflictos interpersonales. Revista Educación y Desarrollo Social / Vol. 11 No. 2, 32-54. DOI: https://doi.org/10.18359/reds.2966 
Amay, A. (2017). Habilidades Sociales. Editorial THGY. https://bit.ly/2RE0wVM

Andueza. (2015). Educar las relaciones interpersonales . España. https://bit.ly/30aK8TX

Arrigoni, F., \& Solans, A. (2018). PROGRAMA DE PROMOCIÓN DE HABILIDADES SOCIALES (PHAS). RUEDES, 65 -85. https://bit.ly/30aK8TX

Astochado, D. y. (2014). Habilidades Sociales. Lima: https://bit.ly/3ce6xlr.

Bandura. (1977). Teoria del aprendizaje social. New Jersey: Englewood. Revista Latinoamericana de Psicología,41(1),109-119. https://bit.ly/3oiYJp7

Bisquerra. (2003). Relaciones Interpersonales. Mexico: Editorial Mac Graw Hill. https://repositorio.unprg.edu.pe/handle/20.500.12893/8638

Buenabad, N., Sánchez, R. ,., Gutiérrez, M., Díaz, A., Ortiz, A., González, T., . . . Villatoro, J. (2020). Cluster Randomized Trial of a Multicomponent SchoolBased Program in Mexico to Prevent Behavioral Problems and Develop Social Skills in Children. Child \& Youth Care Forum; 49, 343-364.DOI: 10.1007/s10566-019-09535-3.

Caballo. (2005). Evaluación de las habilidades sociales. Madrid: Pirámide. https://bit.ly/30aK8TX

Caballo. (2006). Manual de evaluación y entrenamiento de las habilidades sociales. España: Editores Siglo XXI. https://bit.ly/3kk4amb

Caballo, C. O. (2015). España.

Cabello Cuenca, E., Pérez Escoda, N., Ros Morente, A., \& Filella Guiu, G. (2019). Los programas de educación emocional happy 8-12 and happy 12-16. Evaluación de su impacto en las emociones y el bienestar. REOP - Revista Española De Orientación $\quad$ Y $\quad$ Psicopedagogía, 30(2), 53-66. https://doi.org/10.5944/reop.vol.30.num.2.2019.25338

Calderón, D. L. (2015). la relación entre el clima social familiar y las habilidades sociales. Argentina.

Canet-Juric, L., García-Coni, A., Andrés, M. L., Vernucci, S., Aydmune, Y.; Stelzer, F. \& Richard's, M. M. (2020). Intervención sobre autorregulación cognitiva, conductual y emocional en niños: Una revisión de enfoques basados en procesos y en el currículo escolar, en Argentina. Revista Argentina de Ciencias del Comportamiento, 12(1), 1-25. https://doi.org/10.32348/1852.4206.v12.n1.24999 
Carrillo. (2015). Validadción de un programa lúdico para la mejora de las habilidades sociales en niños de 9 a 12 años. [Tesis doctoral,dissertation]. Universidad de España. https://bit.ly/3kk4amb

Casallo, L. (2018). Desarrollo de las habilidades sociales para reducir conductas agresivas. Junín: y [Tesis doctoral,dissertation].,UNIVERSIDAD NACIONAL DE EDUCACIÓN.https://bit.ly/2RHKLgu.

Castro. (2011). Las relaciones interpersonales en la transición de los estudiantes. Revista Electrónica Educare, 193-210.

Castro, G. (2013). La visión del profesorado de Educación Infantil y Primaria de Cantabria sobre la participación y las relaciones interpersonales entre la comunidad escolar . Aula Abierta, 73-84.

Castro, M., \& Ramírez, R. (2019). Aplicación de la psicomotricidad como herramienta de inclusión social en educación infantil. ESHPA - Education, Sport, Health and Physical Activity;3(2)., 23-234.

Chávez, M., \& Aragón, L. (2017). Habilidades sociales y conductas de bullying. Digital Internacional de Psicología y Ciencia Social; 3(1), 21-37.

Combs, S. (2007). Programa de desarrollo de habilidades sociales. Santiago: Universitaria. https://bit.ly/3F3cw9O

Corrales , A., Quijano, N,. \& Góngora , Elías.(2017). Empatía, Comunicación Asertiva Y Seguimiento De Normas. Un Programa Para Desarrollar Habilidades Para La Vida. Enseñanza e Investigación en Psicología,22(1),58-65. https://bit.ly/3H6hvsc

Dagal, A. (2017). Investigation of the Relationship between Communication Skills, Social Competence and Emotion Regulation Skills of Preschool Children in Turkey. Educational Research and Reviews;12 (4), 164-171,DOI: 10.5897/ERR2017.3139.

Diaz, H. (2001). Estrategias docentes para un aprendizaje significativo. Mexico: McGraw-HII. https://bit.ly/3korBLo

Diaz, M. (2017). Programa de estrategias metodológicas participativas para mejorar habilidades sociales en los estudiantes de la ie no 16005 "pedro paulet mostajo"de jaén-2016. Universidad Cesar Vallejo, Chiclayo. Chiclayo: Tesis doctoral dissertation, Universidad Cesar Vallejo,https://bit.ly/2H6SRx1. 
Dominguez, R.(2019). Programa de habilidades sociales en las conductas agresivas de estudiantes de primaria, 2019. [Doctoral dissertation, Universidad Cesar Vallejo]. https://bit.ly/3GYrTIV

Downey, D., \& Gibbs, B. (2020). Kids These Days: Are Face-to-Face Social Skills among American Children Declining?.University of Chicago Press, 125(4), 10301083.https://bit.ly/2RE0wVM

Fernandez, C. (2001). La educacion del ser emocional. Santiago de Chile: Cuarto propio.Índigo. https://bit.ly/31ROX5P

Fernández, L. (2019). Habilidades sociales en la practica docente: una mirada desde los actores de la educacion básica. Revista Electrónica Científica de Investigación Educativa, 4(2), 1303-1315,https://bit.ly/2FNSS8y.

GONZALEZ, A. R. (2004). METODOLOGIA DE LA INVESTIGACION CIENTIFICA. COLOMBIA: Pontifica Universidad Javeriana.

González,R.(2017).Desarrollo de la inteligencia interpersonal e intrapersonal en educación primaria a partir del uso de tecnologías de información y comunicación: estudio de casos. Educación y Tecnología desde una visión Transformadora, 112. https://bit.ly/3EYCcEA

Gutiérrez. (2015). Autoconcepto, dificultades interpersonales, habilidades sociales y conductas asertivas. ISSN electrónico, 42 - 58.

Gutierrez, P. (2015). Autoconcepto, dificultades interpersonales, habilidades sociales y conductas asertivas en adolescentes. España.

Herrera, L., Buitrago, R. E., Cepero, S. (2017). Emotional Intelligence in Colombian Primary School Children. Location and Gender Analysis. Universitas Psychologica, 16(3), xx-xx. https://doi.org/10.11144/Javeriana.upsy16-3.eips

Horna-Clavo,E; Arhuis-Inca,W; Bazalar-Palacios,J(2020) en su artículo científico: Relación de habilidades sociales y tipos de familia en preescolares: estudio de caso Revista Virtual. Fundación Universitaria Católica del Norte Colombia,6,224232.DOI: https://doi.org/10.35575/rvucn.n61a13

Jaramillo, B., \& Guzmán, N. (2019). Las Habilidades Sociales en Ambientes Escolares. Universidad Católica Luis Amigó;(3), 151-162.

Jeffrey. (1982). Evaluación y entrenamiento de las habilidades sociales define a las habilidades sociales. Madrid: Pirámide. https://bit.ly/3qtRiOB 
León, G.,Lacunza, A.(2020).Autoestima y habilidades sociales en niños del Gran San Miguel de Tucumán; Ministerio de Salud de Argentina.Revista Argentina de Salud Pública; 11; 42; 3-2020; 22-31. https://bit.ly/30bhsKR

Linehan. (1984). Efectividad Interpersonal En Situaciones Asertivas. Nueva York: Guilfor Press.

Liwensohn, L. y. (1999). El concepto de habilidad social con especial referencia al comportamiento de las personas deprimidas. Revista de Consultoría y Psicología Clínica, 40, 304-312. https://bit.ly/3F3cw9O

Lopez,L., \& Lopez,B.(2018): El desarrollo de las habilidades socioemocionales como factor influyente en el desempeño académico.UNID sede Tejupilco, México.Revista Atlante: Cuadernos de Educación y Desarrollo (agosto 2018). https://bit.ly/3C2nwTf.

Lorente. (2014). Efecto de la competencia social, la empatía y la conducta prosocial en adolescentes. Valencia .

Mata, S., Gómez, M., \& Dolores, M. (2018). Resolución de problemas interpersonales en niños en exclusión social: Valoración de un programa de entrenamiento. Revista Latinoamericana;50(2), 107-116. https://bit.ly/3c2wDZa

Mendoza, B., \& Maldonado, V. (2017). Acoso escolar y habilidades sociales en alumnado de educación básica. C I E N C I A e r g o -s um; 24(2), 109-116.

Milicic, A. (2011). Climas sociales toxicoa y climas sociales nutritivos para el desarrollo personal en el contexto escolar. . Psykhe, 9. https://bit.ly/3c2wDZa

Monjas, I. (2007). Cómo promover la convivencia: Programa de Asertividad y Habilidades Sociales (PAHS). Madrid: CEPE. Madrid. CEPE. Madrid: CEPE. https://bit.ly/3FhNcNJ

Mooij, B., Fekkes, M., Scholte, R., \& Overbeek, G. (2020). Effective Components of Social Skills Training Programs for Children and Adolescents in Nonclinical Samples: A Multilevel Meta-analysis. Clinical Child and Family Psychology Review; 23, 250-264,doi: 10.1007/s10567-019-00308-x.

Morales, F. (2017). Relaciones entre afrontamiento del estrés cotidiano,autoconcepto, habilidades sociales e inteligenciaemocional. European Journal of Education and Psychology; 10, 41---48. 
Navas. (1991). Cómo controlar su ansiedad en situaciones de evaluación o examen. Santo Domingo: CPE. https://bit.ly/3F363f7

Oliveira,J. Bezerra,T. Souza,P. Ataida,M. Santos,F. \& Santos, A.( 2021).Treinamento em Habilidades Sociais na Pré-escola: uma Revisão de Intervenções Brasileiras. Instituto Superior Politécnico Gaya Psicologia, Educação e Cultura. Brazil .5(1).https://bit.ly/3EMUqsH.

Pena Garrido, M., Extremera Pacheco, N., \& Rey Peña, L. (2017). Las competencias emocionales: material escolar indispensable en la mochila de la vida. Padres Y Maestros / Journal of Parents and Teachers, (368), 6-10. https://doi.org/10.14422/pym.i368.y2016.001

Peñafield. (2010). Habilidades sociales una herramienta para el éxito. España: Editorial Martínez Roca. https://bit.ly/3c2wDZa

Phillips. (1978). The social skills basis of psychopathology. Nueva York: Grune and Stratton. https://bit.ly/2YwHCHk

Porto,M.,Puerta-Morales,L.,Gelves-Ospina,M.,Urrego-Betancourt,Y. (2021). Funciones Ejecutivas y Rendimiento Académico en Educación Primaria de la Costa Colombiana. Electronic Journal of Research in Educational Psychology,19(2), 351-368. https://bit.ly/3kbsCXc.

Pullchs, M. (2018). Programa educativo para la mejora de las habilidades sociales en estudiantes adolescentes. Arequipa: Tesis doctoral, dissertation,UNIVERSIDAD NACIONAL DE SAN AGUSTÍN DE AREQUIPA,https://bit.ly/2ZVuRnk.

Ramírez-Coronel AA., Martínez-Suárez PC, Cabrera-Mejía JB, Buestán-Andrade PA, Torracchi-Carrasco E, Carpio MG.(2020).Habilidades sociales y agresividad en la infancia y adolescencia. Revista AVFT Archivos Venezolanos de Farmacología y Terapéutica, Volumen 39, número 2,https://bit.ly/3n2mq5E

Rebollo, E., \& de la Peña, ,. (2017). Estudio de la Inteligencia Emocional y Función Ejecutiva en Educación. REIDOCREA;6(3), 29-36. https://bit.ly/3c2wDZa

Reyes, M. (2017). Efectos del taller de estrategias comunicativas en las habilidades sociales. Lima: Tesis Docgtoral dissertation,Universidad Cesar Vallejo,https://bit.ly/2RINHcM.

Robert, M. (1978). "Habilidades sociales necesarias en la adolescencia”. España: Pirámide. https://bit.ly/3H4vHSy 
Rodriguez, C. A. (2017). Habilidades de argumentación. Una propuesta para el planteamiento de posibles soluciones a los conflictos interpersonales. Revista Educación y Desarrollo Social / Vol. 11 No. 2, 32-54.

Rodriguez, H. S. (2013). Habilidades para la vida (cognitivas y sociales) en adolescentes de una zona rural. Revista Electrónica de Investigación Educativa, 98-113.

Rodríguez, T. (2013). Variables relacionadas con las habilidades sociales en dos grupos de adolescentes. España. https://bit.ly/3c2wDfr

Rojas. (2011). Manual de programas educativos. Madrid: Pirámide. https://bit.ly/30g16Rs

Rojas Islas, M. E. (2019). Desarrollo de habilidades para la vida. Logos Boletín Científico De La Escuela Preparatoria No. 2, 6(11). https://bit.ly/3H1M7Lz

Romeau. (2015 ). Hacia una teoría personalista de la comunicación interpersonal. Questión, 48.

Rubiales,J., Russo,D., PaneivA,J.(2018). Revisión sistemática sobre los programas de Entrenamiento Socioemocional para niños y adolescentes de 6 a 18 años publicados entre 2011 y 2015. Universidad Nacional de Mar del Plata Consejo Nacional de Investigaciones Científicas y Técnicas, Argentina. Rev. Costarric. Psic vol.37 n.2. http://dx.doi.org/10.22544/rcps.v37i02.05.

Saballs, C. (2002). Formación del profesorado orientada al desarrollo de competencias de gestión del aula de ESO; el afrontamiento de situaciones críticas. Interuniv, 46. https://bit.ly/3c2wDZa

Salvador. (2017). Relaciones interpersonales en el proceso de enseñanza aprendizaje, un reto para la inclusión. Revista Conrado, 146-150. https://bit.ly/2YwHCHk

Tapia, C., \& Cubo, S. (2017). Habilidades sociales relevantes:. Revista Internacional de Investigación en Educación; 9(19)., 133-148. https://bit.ly/3ww2pHJ

Vásquez, P. (2018). Efecto de un programa de tutoría socio afectiva en la. Lima: Tesis doctoral,dissertation,Universidad Cesar Vallejo,https://bit.ly/35SHNxY.

Vigotsky. (1997). Programa de habilidades sociales . Salamanca: Amaru. https://bit.ly/31MVUF8

Wolpe. (1958). Psicoterapia Por Inhibición Recíproca. California : Stanford University Press. https://bit.ly/3ww2hyk 
Yogman, M., Garner, A., Hutchinson, J., \& Hirsh, K. (2018). COMMITTEE ON PSYCHOSOCIAL ASPECTS OF CHILD AND FAMILY HEALTH and COUNCIL ON COMMUNICATIONS AND MEDIA. Pediatrics142 (3).

Zhang, X., Ying Hu, B., Ren, L., Huo, S., \& Wang, M. (2019). An action research study in an Icelandic preschool: Developing consensus about values and values education. Revista internacional de la primera infancia; 48, 161 - 177,DOI: 10.1002 / cad.20271 